\title{
Conditions-cadres générales et explications relatives au contrat de travail
}

Etant donné que, depuis 1991, les sociétés cantonales de médecine élaborent leurs propres recommandations de salaire pour les assistantes médicales, nous renonçons à publier des recommandations salariales pour l'ensemble de la Suisse.

Les conditions-cadres ci-après conservent toutefois leur valeur pour fixer individuellement le salaire d'une assistante médicale.

\section{Formation}

- Diplôme de la Fédération des médecins suisses ou certificat de capacité de la FMH avec certificat de radioprotection et autorisation de faire des radiographies;

- Certificat fédéral de capacité d'assistante médicale.

\section{Conditions de travail: bases de calcul}

- semaine de 42 heures en moyenne annuelle;

- 4 semaines de vacances (assistantes médicales de moins de 20 ans et de plus de 50 ans: 5 semaines).

Toute dérogation importante à ces conditions sera répercutée sur le salaire.

\section{Normes régionales}

Les salaires doivent être adaptés aux conditions régionales.

\section{4. $13^{\mathrm{e}}$ salaire}

Un $13^{\text {e }}$ salaire doit être versé à l'assistante médicale à la fin de l'année. Si les rapports de service n'ont pas duré pendant toute l'année civile, le $13^{\mathrm{e}}$ salaire sera versé au prorata des mois d'engagement.

\section{Primes d'ancienneté et augmentations}

\section{du salaire réel}

Le montant d'une prime d'ancienneté devrait figurer, également en 2012, parmi les points discutés au cours de l'entretien annuel sur les qualifications de l'employée. Au moment de définir le salaire, il convient notamment de prendre aussi en considération toute éventuelle fonction supplémentaire (par ex. formation des personnes en apprentissage).

Correspondance: FMH/Assistantes médicales Elfenstrasse 18 CH-3000 Berne 15 Tél. 0313591111 Fax 0313591112

\section{Compensation du renchérissement} (www.bfs.admin.ch)

Le renchérissement devrait être compensé. Lorsque la compensation du renchérissement est convenue par contrat, elle doit être accordée en tous les cas. Si le renchérissement est négatif, il ne doit pas être compensé (ISPC septembre 2011: 0,5\%; octobre 2011: -0,1\%, indice de base: décembre 2010).

\section{Travail à temps partiel rétribué au mois}

Pour le travail à temps partiel, le salaire brut recommandé s'élève au $1 / 42$ du salaire brut entier multiplié par le nombre d'heures de travail hebdomadaire convenu (base de calcul: 42 heures hebdomadaires).

\section{Salaire horaire}

Un salaire horaire est recommandé en cas d'horaire de travail très réduit et en même temps irrégulier. Le taux horaire conseillé est de $6 \%$ du salaire mensuel pour une activité à temps complet (le $13^{\text {e }}$ salaire y étant inclus au pro rata). Il sera complété par une contribution aux vacances de $8,33 \%$ correspondant aux quatre semaines de vacances habituelles (pour 5 semaines le taux sera de $10,64 \%$ et pour 6 semaines 13,04\%, etc.). Ce mode de calcul est également valable pour le paiement des heures supplémentaires.

\section{Heures supplémentaires}

\section{(chiffre 2 du contrat type)}

Dans la mesure du possible, les heures supplémentaires doivent être compensées par des vacances ou du temps libre d'une même durée. Si ce n'est pas possible, on peut désormais choisir parmi les variantes suivantes:

- L'indemnisation des heures supplémentaires par une majoration de salaire de $25 \%$ pour une activité à temps complet ou à temps partiel (préconisée par la FMH et la FSAAM).

- Dans le cas d'une activité à temps partiel, les heures supplémentaires sont indemnisées selon le taux horaire normal dans la mesure où elles ne dépassent pas la durée du temps de travail réglementaire d'une assistante à temps plein au cabinet médical (préconisée par la SVA);

- D'un commun accord écrit, l'employeur et l'assistante médicale peuvent choisir une autre réglementation.

Le taux horaire est de 6\%o, d'un plein salaire mensuel pour une activité à temps complet, auquel s'ajoute la 


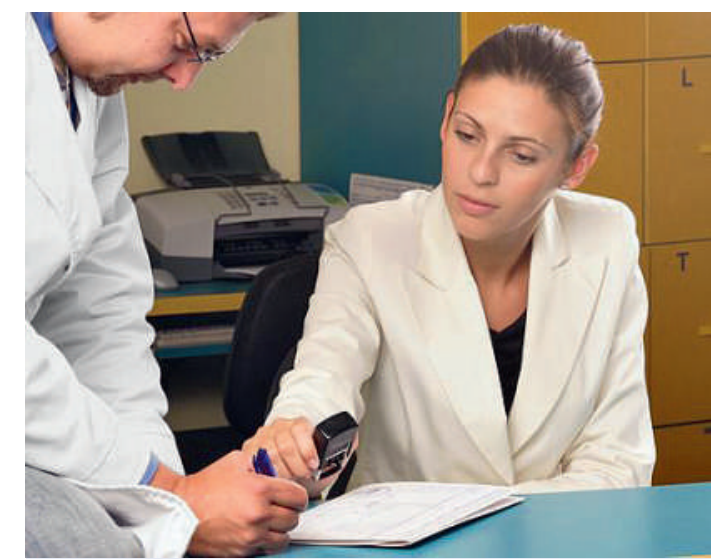

Le montant d'une prime d'ancienneté devrait figurer, également en 2012, parmi les points discutés au cours de l'entretien annuel sur les qualifications de l'employée.

contribution aux vacances (cf. chiffre 8, «salaire horaire»).

\section{Les dispositions et recommandations sui- vantes s'appliquent à tous les salaires:}

1. Déductions sur le salaire brut

- AVS, AI, APG, ACI: 6,25\% (AVS, AI, APG = 5,15\%, $\mathrm{ACI}=1,1 \%)$;

- Assurance-accidents non professionnels (pour un engagement de plus de 8 heures par semaine): déduction selon le contrat d'assurance conclu;

- Prévoyance professionnelle ( $2^{\mathrm{e}}$ pilier LPP): part de l'employée à la contribution (habituellement $50 \%$ ) calculée en fonction de l'âge, selon le certificat d'assurance.

\section{Contrats de travail et autres conditions} d'engagement

Nous préconisons d'établir le contrat par écrit, sur la formule de la FMH créée conjointement avec les associations des assistantes médicales, accompagnée des recommandations cantonales. Ces documents peuvent être obtenus aux adresses suivantes:
- Fédération des médecins suisses FMH, Elfenstr. 18, case postale 170, 3000 Berne 15, tél. 03135911 11, www.fmh.ch, mpa@fmh.ch

- Association romande des assistantes médicales (ARAM), case postale 5263, 1002 Lausanne, tél. 0793801244 / 07743679 27, www.aram-vd.ch; info@aram-vd.ch

- Association suisse des assistantes médicales (SVA), case postale 6432, 3001 Berne, tél. 0313805454 www.sva.ch, sekretariat@sva.ch

- Berufsverband Medizinischer Praxisassistentinnen (BMPA), Obergrundstr. 65, 6003 Lucerne, tél. 04131011 21, www.bmpa.ch, sekretariat@ bmpa.ch

- Fédération suisse des associations d'assistantes médicales (FSAAM), chemin du Lussy 18, 1806 St-Légier, tél. 02194345 64, www.fsaam.ch, fsaam-ch@ bluewin.ch

- Association genevoise des assistantes médicales (AGAM), Case postale 18, 1241 Puplinge, www. agam-ge.ch, presidente@agam-ge-ch

L'Association des assistantes médicales du Tessin a sa propre convention de travail ou contrat de travail; son adresse est la suivante:

- Associazione Ticinese Assistenti di studio Medico (ATAM), c/o M. Carrera, Via dei Faggi 2 A, 6912 Lugano-Pazzallo,www.atam.ch, atam@bluewin.ch

Les salaires des personnes en formation se basent sur les recommandations cantonales.

Délégués aux questions concernant les assistantes médicales: Dr Adrian Sury, président /

Dr Renato Tognina, vice-président

Pour la Fédération suisse des associations des assistantes médicales FSAAM (AGAM, ARAM, ATAM, BMPA):

Mme Elwina Kaufmann, présidente

Pour l'Association suisse des assistantes médicales SVA: Me Bruno Gutknecht, secrétaire central 